\title{
Mechanisms of LRRK2-Mediated Neurodegeneration
}

\author{
Elpida Tsika • Darren J. Moore
}

Published online: 24 March 2012

(C) Springer Science+Business Media, LLC 2012

\begin{abstract}
Mutations in the leucine-rich repeat kinase 2 (LRRK2) gene represent the most common cause of familial Parkinson's disease (PD), whereas common variation at the LRRK2 locus is associated with an increased risk of idiopathic PD. Considerable progress has been made toward understanding the biological functions of LRRK2 and the molecular mechanisms underlying the pathogenic effects of disease-associated mutations. The development of neuronal culture models and transgenic or viral-based rodent models have proved useful for identifying a number of emerging pathways implicated in LRRK2-dependent neuronal damage, including the microtubule network, actin cytoskeleton, autophagy, mitochondria, vesicular trafficking, and protein quality control. However, many important questions remain to be posed and answered. Elucidating the molecular mechanisms and pathways underlying LRRK2-mediated neurodegeneration is critical for the identification of new molecular targets for therapeutic intervention in PD. In this review we discuss recent advances and unanswered questions in understanding the pathophysiology of LRRK2.
\end{abstract}

Keywords Parkinson's disease · PARK8 - LRRK2 . Parkinsonism · Neuronal toxicity · Animal models · Neurite outgrowth $\cdot$ Protein kinase $\cdot$ Microtubules $\cdot$ Protein aggregation $\cdot$ Autophagy $\cdot$ Neuronal cell death . Neurodegeneration

E. Tsika $\cdot$ D. J. Moore $(\triangle)$

Laboratory of Molecular Neurodegenerative Research, Brain Mind Institute, School of Life Sciences,

Ecole Polytechnique Fédérale de Lausanne (EPFL), SV-BMI-LMNR, AI 2150, Station 15,

1015 Lausanne, Switzerland

e-mail: darren.moore@epfl.ch

\section{Introduction}

Parkinson's disease (PD) is the most common neurodegenerative movement disorder affecting $1 \%$ to $2 \%$ of the population above 60 years of age [1]. Motoric abnormalities result from the profound degeneration of distinct neuronal populations within the brain, most prominently the dopaminergic neurons of the substantia nigra and their striatal projections resulting in a dramatic reduction of dopamine content in the caudate putamen [2,3]. Neurodegeneration is typically accompanied by the presence of proteinaceous intracytoplasmic inclusions termed Lewy bodies in surviving neurons that are enriched with $\alpha$-synuclein protein. Most PD cases occur in a sporadic manner although 5\% to $10 \%$ of cases are inherited with mutations identified in several genes that clearly segregate with disease in families with PD. Although familial PD is relatively rare, understanding the function of mutated gene products that influence disease susceptibility provides a unique opportunity to elucidate the molecular pathogenic mechanisms and pathways underlying neuronal degeneration and to develop disease-modifying or neuroprotective therapies.

Mutations in the leucine-rich repeat kinase 2 (LRRK2, PARK8, OMIM 607060) gene cause late-onset autosomaldominant PD [4, 5], and represent the most common known cause of familial PD. Recent genome-wide association studies also suggest that common variation at the LRRK2 locus influences the risk of developing sporadic PD [6, 7]. Among numerous putative pathogenic variants identified to date, at least six missense mutations in the LRRK2 gene are known to clearly segregate with disease (ie R1441C, R1441G, R1441H, Y1699C, G2019S, and I2020T) [8], indicating that they represent authentic disease-causing variants. The most frequent LRRK2 mutation, G2019S, has been identified in up to about $40 \%$ of familial PD cases depending on 
ethnicity, and has been detected in apparent sporadic PD cases. The latter observation can most likely be attributed to the incomplete penetrance of the G2019S variant, which ranges from $28 \%$ at 59 years to $74 \%$ at 79 years [9].

The clinical features of LRRK2 mutation carriers are virtually indistinguishable from sporadic PD [9]. Neuropathological data indicate that the majority of LRRK2 mutation carriers display typical brainstem Lewy body pathology although there are reported cases that exhibit either tau-positive neurofibrillary tangle pathology, ubiquitin-positive inclusions, or the distinct absence of inclusion pathology [5,10-12]. This heterogeneous pathology of LRRK2 PD brains might suggest that LRRK2 can participate in the regulation of protein aggregation pathways that are common to a number of neurodegenerative diseases. The lack of protein inclusion pathology in certain LRRK2 PD brains, although rare, might also indicate that neuronal degeneration can occur independently from inclusion formation. The significance of $L R R K 2$-related heterogeneous neuropathology remains to be clarified.
In this review, we discuss recent advances in understanding the physiological function of LRRK2 and the potential mechanisms through which disease-associated mutations precipitate selective neuronal dysfunction and degeneration in PD.

\section{Structure and Cellular Localization of LRRK2}

LRRK2 is a multi-domain protein of 2527 amino acids that belongs to the ROCO family of proteins. ROCO proteins contain a characteristic Ras-of-Complex (ROC) GTPase domain adjacent to a C-terminal-of-ROC (COR) linker region. LRRK2 also contains several distinctive domains including a serine/threonine protein kinase domain and putative protein-protein interaction domains flanking the central ROC-COR-kinase catalytic region: LRRK2specific, ankyrin, and leucine-rich repeat motifs in the $\mathrm{N}$ terminal region and WD40 repeats near the C-terminus of the protein (Fig. 1) [13]. The existence of multiple protein-

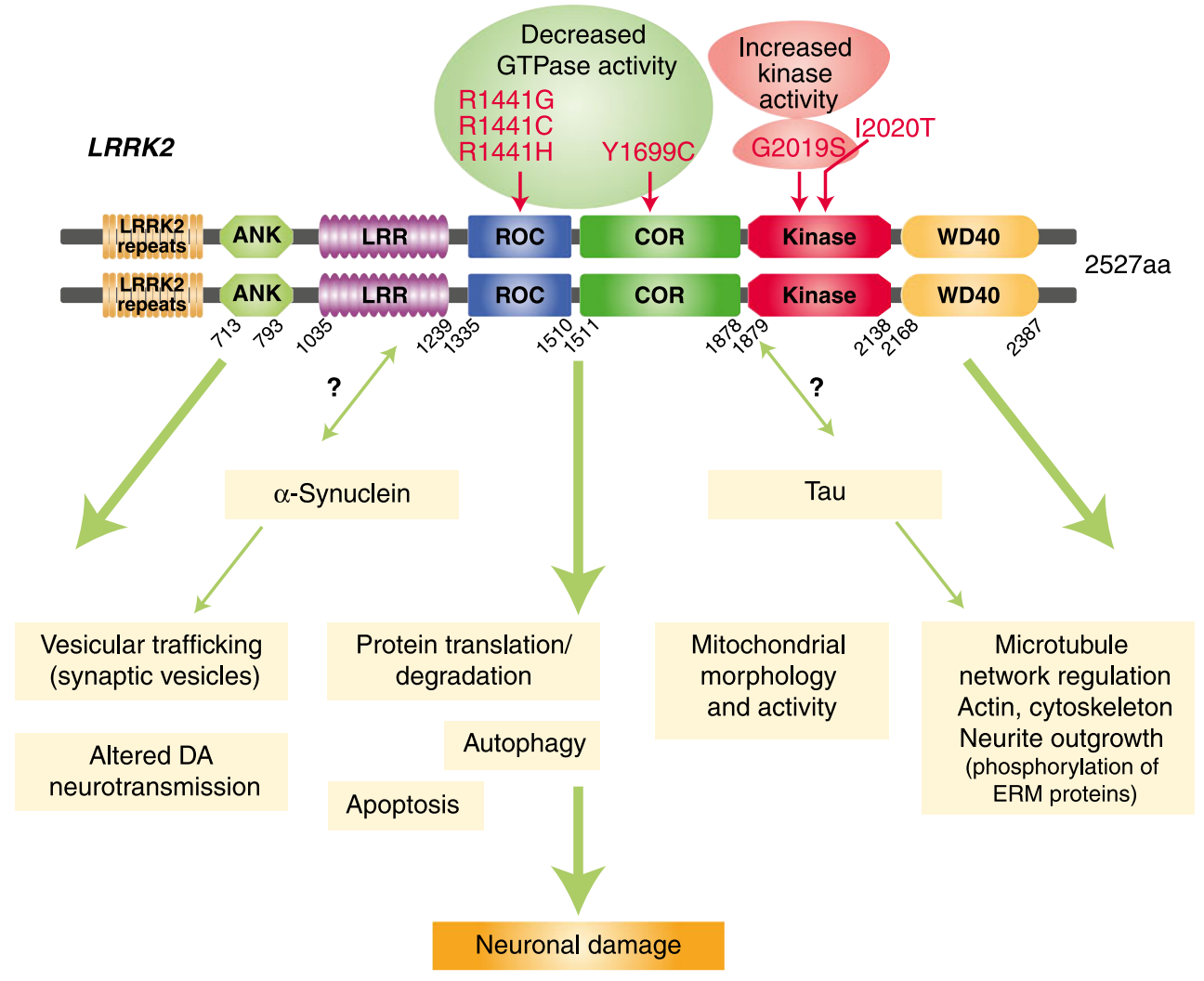

Fig. 1 LRRK2 is a dimeric multi-domain protein consisting of protein-protein interaction domains surrounding a catalytic core (ROC-COR-kinase) where the most common pathogenic mutations are located (as indicated). Pathogenic mutations in LRRK2 lead to decreased GTPase activity (ie, R1441C/G/H or Y1699C) or increased kinase activity (ie, G2019S). The development of cellular and animal models has shed light on LRRK2-related molecular pathways. LRRK2 has been implicated in regulating the microtubule network, actin cytoskeleton, neurite outgrowth, autophagy-lysosomal pathway, apoptosis, mitochondrial morphology and activity, vesicular trafficking (ie, synaptic vesicle exocytosis/endocytosis), dopaminergic (DA) neurotransmission, protein translation, and degradation pathways, which could potentially underlie neuronal damage. The potential functional interaction of LRRK2 with $\alpha$-synuclein and tau as well as the molecular mechanisms through which disease-associated mutations lead to neurodegeneration require further elucidation. ERM — ezrin/radixin/moesin 
protein interaction domains could imply that LRRK2 may act as a scaffold for the assembly of protein complexes, some of which could regulate or be modified by the enzymatic domains of LRRK2 following their recruitment. While LRRK2 interacts with a myriad of proteins, the identification of a common LRRK2 protein complex has not been forthcoming and is likely influenced by the cellular milieu and/or subcompartment. Similar to other protein kinases, LRRK2 normally exists as a dimeric structure minimally mediated via its ROC domain and dimerization is required for its kinase activity and localization to cellular membranes [14-17]. The WD40 domain also appears to be important for dimerization and kinase activity [18].

LRRK2 is widely expressed in many tissues and cell types [19]. LRRK2 expression is first detected in the rodent brain by embryonic day 16 to 17 with increasing expression during neuronal maturation and postnatal stages, whereas in adult rodents LRRK2 mRNA is expressed at highest levels in kidney, lung, and lymph nodes [20-25]. Notably, deletion of LRRK2 in mice induces cytopathological abnormalities in kidney and lung tissues [26-28]. In the mammalian brain, LRRK2 mRNA and protein is detected at low levels in dopaminergic neurons of the substantia nigra and ventral tegmental area while it is abundant in dopamine-innervated areas such as the striatum and cerebral cortex as well as in the cerebellum and hippocampus [8, 21-23, 29]. LRRK2 protein is localized predominantly to neuronal cells under normal conditions where it is present throughout the cytoplasm associated with various intracellular membranes and vesicular structures as revealed by confocal and electron microscopy, and subcellular fractionation [20]. In particular, LRRK2 localizes to endosomes, lysosomes, multivesicular bodies, mitochondrial outer membrane, lipid rafts, microtubule-associated vesicles, the Golgi complex, and the endoplasmic reticulum [20, 30-32]. LRRK2 is also enriched in synaptosomal fractions from rodent brain [20]. The subcellullar localization of LRRK2 provides clues for its physiological function(s) within neurons, for example, participation in cytoskeletal organization and vesicular transport, as will be discussed below. The association of LRRK2 with intracellular membranes represents a potential mechanism for the regulation of its dimerization and enzymatic activity [14], reinforcing a role for LRRK2 in membrane dynamics and transport.

\section{LRRK2 Enzymatic Activity: A Functional GTPase and Kinase?}

The kinase domain of LRRK2 shares closest sequence homology to receptor-interacting protein kinases (RIPKs) and to mixed-lineage kinases (MLKs), a subclass of the mitogenactivated protein kinase kinase kinase (MAPKKK) family.
LRRK2 does not appear to directly participate in MAPK signaling in mammalian cells but can phosphorylate the stress-activated MAP kinase kinase proteins, MKK3, 4, 6, and 7, in vitro [33-36]. Most studies of LRRK2 kinase activity rely on in vitro autophosphorylation assays although it is not yet clear whether autophosphorylation directly correlates with the intrinsic activity of LRRK2 [36-38]. In vitro, LRRK2 can phosphorylate the generic kinase substrate, myelin basic protein [37, 38], as well as two pseudosubstrate peptides, LRRKtide and Nictide, based upon phosphorylation of Thr558 of moesin [37, 39]. However, despite substantial in vitro evidence demonstrating the kinase activity of LRRK2, there is currently lack of evidence for LRRK2-mediated phosphorylation occurring in mammalian cells or tissues. A number of putative substrates for LRRK2 have been identified mostly in vitro, including ezrin/radixin/moesin (ERM) (Thr558) [37], $\beta$-tubulin (Thr107) [40], FoxO1 (Ser319) [41], 4E-BP1 (Thr37/46) [42], MKK proteins [33], $\alpha$ synuclein (Ser129) [43], and Drosophila Futsch [44], but these observations have not yet been independently verified especially in mammalian cells or tissues [39, 45]. Efforts to identify robust LRRK2 substrates in mammalian cells and tissues by SILAC (stable isotope labeling by amino acids in cell culture) or other phosphoproteome profiling methods are presently being conducted in many laboratories, including our own, and may offer further insight into the significance of LRRK2 kinase activity. At this juncture, the most robust substrate for LRRK2 appears to be itself where it autophosphorylates numerous sites in or around the GTPase domain, some of which have been shown to regulate enzymatic activity [46-49]. It remains to be clarified whether kinase activity simply serves to autoregulate LRRK2 activity and/or whether bona fide substrates exist for LRRK2, in particular mammalian tissues, cells, and subcellular compartments.

The GTPase domain of LRRK2 shares homology to members of the Rab GTPase family. LRRK2 can bind GTP via its guanine nucleotide phosphate-binding (P-loop) motif and can hydrolyze bound GTP in vitro albeit with low activity [36, 50-53]. The presence of two enzymatic domains suggests the potential for an intrinsic regulatory mechanism of LRRK2 activity with parallels to the classic Ras/Raf signal cascade. Initial studies demonstrated that GTP binding enhanced the kinase activity of LRRK2, whereas abolishing GTP binding via P-loop null mutations (ie, T1348N) critically impaired kinase activity [36, 52, 53]. ROC domain mutations that enhance GTP hydrolysis also impair kinase activity [52]. A recent study has revealed that LRRK2 kinase activity is most likely independent of GTP binding per se but instead depends on the capacity for GTP binding, which may indicate the requirement for an upstream guanine nucleotide-binding protein for the GTPdependent activation of LRRK2 [54]. While GTPase activity does not appear to critically require a functional kinase 
domain $[36,52,53]$, autophosphorylation within the GTPase domain may serve a regulatory function [47-49], which implies a complex yet poorly understood bidirectional relationship between the GTPase and kinase domains of LRRK2. This concept of intramolecular regulation of LRRK2 activity may provide an explanation for how spatially distinct mutations that influence either GTPase or kinase activity can commonly precipitate neurodegeneration in PD. The activity of small GTPases is typically regulated by guanine nucleotide exchange factors (GEFs), which promote the exchange of GDP for GTP leading to activation, and GTPase-activating proteins (GAPs), which promote the hydrolysis of bound GTP leading to inactivation. Accordingly, a putative GEF, ARHGEF7 [55], and GAP, ArfGAP1 [56], for LRRK2 have recently been identified. ArfGAP1 interacts with LRRK2 in vitro and in vivo and promotes its GTP hydrolysis activity but also serves as a robust substrate of LRRK2-mediated phosphorylation in vitro [56]. The absence of these regulatory factors from previous studies may explain why LRRK2 appears to exhibit poor GTPase activity in vitro.

\section{Disease-Associated LRRK2 Mutations: Common Versus Distinct Pathogenic Effects}

The majority of familial disease-associated mutations tend to cluster within the central ROC-COR-kinase region of LRRK2 [8, 57]. The dominant inheritance pattern of missense mutations and the general absence of deletion or truncation mutations reported to date provide some support for a toxic gain-of-function mechanism rather than a loss-offunction mechanism (ie, dominant-negative or haploinsufficient effects) underlying the pathogenic effects of mutations. Clarifying the mechanism of action of familial mutations and in particular their common pathogenic properties has important implications for the development of appropriate therapeutic strategies. For LRRK2 mutations, there is as yet no consensus on a common pathologic effect. In almost all functional assays reported, familial mutations do not obviously manifest a loss-of-function phenotype and are equally if not more active than wild-type LRRK2.

The G2019S and I2020T mutations are located within the activation loop of the kinase domain, a highly conserved region that is critical for substrate access, where only the G2019S variant robustly enhances kinase activity in vitro $[38,57,58]$. While these observations may implicate enhanced kinase activity in the pathogenic actions of familial mutations, it is worth remembering that other mutations located within the ROC-COR-kinase region do not consistently alter kinase activity across multiple independent studies [59]. This might suggest that kinase activity is not likely to be the major pathogenic output of LRRK2, unless one assumes that distinct mutations promote neuronal damage through distinct rather than common mechanisms. Mutations located within or near the GTPase domain (ie, R1441C, R1441G, and Y1699C) tend to modestly impair GTP hydrolysis activity, which could lead to a prolonged activation of LRRK2 or a persistent interaction with an unknown GTP-dependent interacting partner $[50,51,60$, 61]. The G2019S and I2020T mutations do not alter GTP hydrolysis activity but could conceivably alter LRRK2 autophosphorylation at sites within the GTPase domain and thus activity [60]. At present, common alterations in enzymatic activity are not sufficient to explain the pathogenic effects of LRRK2 mutations.

Most evidence for the pathogenic actions of LRRK2 mutations exists for the common G2019S variant. Overexpression of the G2019S mutant (as well as wild-type and R1441C) in cultured primary neurons or neuroblastoma cell lines induces variable amounts of apoptotic cell death and genetic or pharmacological inhibition of kinase activity attenuates cell death $[36,53,62-64 \cdot, 65]$. This may suggest that LRRK2-mediated phosphorylation of its substrates and/or itself are implicated in pathways leading to neuronal damage. The nature of this pathway remains elusive. It has not yet been possible to clarify why inhibition of the kinase activity of the R1441C mutant, which does not consistently exhibit enhanced kinase activity, prevents LRRK2-induced neuronal cell death [53, 63, 65]. Genetic inhibition of GTPase activity by preventing GTP binding also blocks neuronal damage induced by wild-type and G2019S LRRK2 [36, 53]. Kinase and GTPase activity may therefore represent useful molecular targets for interfering with LRRK2-mediated neuronal damage although the mechanism of action is not clearly understood and these effects have not been subjected to rigorous validation in vivo in animal models.

The pathogenic effects of the G2019S mutation have also been demonstrated to some extent in animal models. In Drosophila, the transgenic expression of human G2019S LRRK2 induces the adult-onset selective loss of dopaminergic neurons and locomotor dysfunction [66], which could be attenuated by inhibition of LRRK2 kinase activity [67], or by overexpression of parkin [68], a gene product associated with autosomal-recessive PD. Similarly, the delivery of human adenoviral vectors expressing G2019S LRRK2 to the striatum of adult rats leads to retrograde dopaminergic axonal transport of LRRK2 and the progressive loss of nigral dopaminergic neurons [69•]. Intriguingly, dystrophic dopaminergic neurites containing hyperphosphorylated tau are also produced by wild-type and G2019S LRRK2 expression in this adenoviral model, which suggests that neuronal degeneration can be dissociated from protein inclusion pathology [69•].

A second gene transfer model based on intrastriatal delivery of herpes simplex virus amplicon vectors expressing 
G2019S LRRK2 to adult mice induces dopaminergic neurodegeneration whereas genetic and pharmacological inhibition of LRRK2 kinase activity attenuate this neuronal loss [64•]. Kinase inhibition may offer a promising therapeutic strategy for preventing LRRK2-dependent neurodegeneration but these studies await independent confirmation using newer highly selective inhibitors of LRRK2 kinase activity $[63,70]$. While viral-based models have proved successful in reproducing mutation-dependent, LRRK2-mediated neuronal degeneration, transgenic mice overexpressing human G2019S LRRK2 driven by the platelet-derived growth factor- $\beta$ promoter also develop age-dependent nigral dopaminergic neuronal loss together with the accumulation of autophagic vacuoles and mitochondrial abnormalities but with the distinct absence of classical protein inclusions or aggregates [71•]. Similarly, BAC transgenic mice overexpressing R1441G LRRK2 develop progressive levodoparesponsive motor deficits, reduced striatal dopamine release, and dopaminergic axonal pathology containing hyperphosphorylated tau but in the absence of frank nigral neuronal loss [72•].

A number of LRRK2 transgenic mouse models exhibit impaired or abnormal dopaminergic neurotransmission, including R1441G and G2019S BAC transgenic mice and R1441C knockin mice, revealing a potential role for LRRK2 in the nigrostriatal dopaminergic pathway [72•, 73-75]. Impaired dopaminergic neurotransmission manifests in the absence of overt nigral neuronal loss or axonal degeneration in the striatum of LRRK2 BAC mice [72•,73], which could suggest that altered neurotransmission may relate to the involvement of LRRK2 in regulating synaptic vesicle trafficking and localization or could represent an early event prior to neuronal loss [25, 60, 76]. LRRK2 normally controls the storage and mobilization of synaptic vesicles within the presynaptic recycling pool [25], and LRRK2 overexpression disrupts synaptic vesicle exocytosis and endocytosis [60, 76]. Similarly, deletion of the LRRK2 homolog, lrk-1, in Caenorhabditis elegans regulates the axonal-dendritic polarity of synaptic vesicle protein transport via sorting at the Golgi apparatus [77]. It will be important to clarify the effects of LRRK2 mutations on nigrostriatal dopaminergic neurotransmission in rodent models and whether this is due to synaptic pathology or to abnormal regulation of synaptic vesicle transport and recycling within dopaminergic neurons.

Another common effect induced by LRRK2 mutations is the induction of neuronal cell death pathways. Initially, the R1441C, Y1699C, and G2019S mutants were shown to promote neuronal cell death through the intrinsic mitochondrial-dependent apoptotic cell death pathway involving nuclear condensation, mitochondrial cytochrome $c$ release, and caspase- 3 activation $[62,65,78]$. Apoptotic cell death could be blocked by caspase- 3 inhibition and by deletion of Apaf-1, which is required for apoptosome formation and caspase activation [78]. Activated caspase-3 has also been observed in rat dopaminergic neurons transduced via intranigral delivery of AAV2 vectors expressing the G2019S but not wild-type LRRK2 kinase domain [79]. Since the isolated kinase domain of LRRK2 is not known to be functional [37], cell death in this model most likely occurs in a kinase-independent manner. LRRK2 mutations have also been shown to enhance the interaction with the death adaptor Fas-associated protein with death domain (FADD), which mediates the recruitment and activation of caspase- 8 required for the extrinsic cell death pathway [80]. Mutant LRRK2-dependent neuronal death is dependent on FADD and caspase- 8 [80]. It will be important to assess whether apoptotic markers, including caspases, are induced in rodent models expressing full-length mutant LRRK2 that exhibit neurodegeneration and which cell death pathways, intrinsic and/or extrinsic, are potentially involved. Inhibition of apoptotic pathways as a strategy for preventing LRRK2dependent neuronal damage warrants further validation in animal models.

\section{Molecular Pathways Implicated in LRRK2-Induced Neuronal Damage}

LRRK2 has so far been implicated in diverse molecular pathways and cellular functions, such as vesicular trafficking, neurite outgrowth, cytoskeletal regulation, autophagy, mitochondrial function, and translational control (Fig. 1). The localization of LRRK2 to intracellular vesicles and membranous compartments in neurons and the presence of kinase and GTPase enzymatic domains provide potential clues to its physiological function. For example, microtubule dynamics and neuronal polarization as well as axon growth are known to be regulated by signaling pathways controlled by kinases and small GTPases [81]. Accordingly, LRRK2 plays a role in regulating the length and complexity of neuronal processes.

Neurite Morphology, Actin Cytoskeleton, and Microtubules

Overexpression of G2019S or I2020T LRRK2 robustly reduces neurite length and branching in neural cell lines and cultured primary neurons [71•, 79, 82, 83•], whereas the effects of other familial mutations are less prominent. Oppositely, ablation of LRRK2 expression promotes neurite length and complexity [79, 83•]. Kinase activity appears to be required for LRRK2-induced neurite shortening [63, 79]. One potential mechanism for regulating neurite morphology is through the LRRK2-mediated phosphorylation of proteins that regulate microtubule assembly, cytoskeletal organization, and axonal growth. The ERM protein family was originally 
identified as putative substrates of LRRK2 kinase activity in vitro [37], and it was subsequently shown that ERM protein phosphorylation by LRRK2 occurring in neuronal filopodia promotes rearrangement of the actin cytoskeleton and impairs the neurite outgrowth of developing neurons $[37,83 \bullet]$. It is not yet clear whether ERM proteins represent direct or indirect substrates of LRRK2-mediated phosphorylation within neurons [83•]. The effects of G2019S LRRK2 on neurite morphology can be rescued by overexpression of the Rho GTPase, Rac1 [84], which regulates actin cytoskeleton remodeling, and by inhibition of the GTPase-activating protein, ArfGAP1 [56], which functions as a GAP for LRRK2 and is involved in the dissociation of coat proteins from intracellular vesicles. Autophagy also appears to be required for the effects of G2019S LRRK2 on neurite morphology [82].

LRRK2 can also regulate the microtubule network. LRRK2 was shown to physically interact with $\alpha$ - and $\beta$-tubulin via its GTPase domain and can phosphorylate brain-derived $\beta$-tubulin isoforms at Thr107 and increase microtubule stability in vitro, although $\beta$-tubulin phosphorylation has not yet been confirmed in vivo $[40,85]$. Overexpression of wild-type or G2019S LRRK2 in transgenic mice impairs microtubule assembly consistent with enhanced tubulin polymerization [86••]. Intact kinase activity and the WD40 domain of LRRK2 may be required for the association with microtubules [87]. A recent study suggests that G2019S LRRK2 expression may cause dendrite degeneration in Drosophila by promoting the phosphorylation of the microtubule-associated protein tau at Thr212 by the GSK3 $\beta$ homolog Shaggy, which induces tau hyperphosphorylation and its mislocalization to dendrites [44]. The abnormal accumulation, processing, or hyperphosphorylation of tau also occurs in vivo in LRRK2 PD brains and in certain transgenic and viral-based rodent models expressing R1441C, R1441G, or G2019S LRRK2 [5, 69•, 72•, 73, 74, 79, 88], which may reflect perturbed microtubule assembly. LRRK2 may therefore regulate the organization of the actin cytoskeleton and microtubule assembly by direct or indirect signaling to some of its protein components, which may underlie the effects of LRRK2 on neurite morphology. It is not yet clear whether the effects of LRRK2 mutations on neurite outgrowth reflect neuronal toxicity that occurs prior to neuronal cell death, or whether they reflect a physiological function of LRRK2 in regulating neurite morphology. That LRRK2 ablation oppositely enhances neurite outgrowth could argue in favor of the latter mechanism. Nevertheless, neurite phenotypes provide a sensitive measure of LRRK2 activity and may be useful for identifying genetic modifiers or defining the efficacy of small molecule LRRK2 inhibitors.

\section{Inclusion Formation and Abnormal Autophagy}

PD brains harboring LRRK2 mutations exhibit heterogeneous protein inclusion pathology, including Lewy bodies, neurofibrillary tangles, and ubiquitin deposits, although the majority of cases manifest typical brainstem $\alpha$-synucleinpositive Lewy body pathology $[10,11]$. The contribution of LRRK2 to the formation of intraneuronal protein inclusions is not yet clear. In general, LRRK2 does not appear to be a common component of Lewy bodies or other protein inclusions based on studies using validated antibodies [19, 20, $22,65,89]$, suggesting that it is not likely to be a core structural component of such inclusions. LRRK2 could potentially modulate pathways that regulate protein accumulation and/or aggregation, such as the ubiquitinproteasomal system (UPS), the autophagy-lysosomal pathway, molecular chaperones, protein translation, axonal or microtubule transport, or vesicular trafficking. A recent study suggests that LRRK2 may cause a general impairment of proteasomal degradation leading to the accumulation of proteasomal substrates including $\alpha$-synuclein [90]. However, the mechanism underlying $\alpha$-synuclein aggregation in LRRK2 PD brains is not known. What is also not yet known is whether $\alpha$-synuclein (or tau) aggregation is critically required for mediating LRRK2-dependent neurodegeneration or instead represents a benign effect secondary to neuronal dysfunction. The application of viral vectors expressing LRRK2 mutants to $\alpha$-synuclein or tau knockout mice, or crossbreeding studies of appropriate mouse models, will be important for clarifying their contribution to dopaminergic neuronal degeneration.

A recent study has attempted to evaluate the pathophysiological interaction of LRRK 2 and $\alpha$-synuclein in vivo in transgenic mice. G2019S LRRK2 expression enhances the abnormal somatic accumulation of $\alpha$-synuclein and neuropathology that develop in forebrain neurons of A53T $\alpha$ synuclein transgenic mice whereas LRRK2 deletion reduces these phenotypes [86••]. Whether LRRK2 and $\alpha$-synuclein functionally interact in nigrostriatal dopaminergic neurons of mice is not yet known. Potential mechanisms for the modulatory actions of LRRK2 include impaired Golgi complex integrity, perturbed microtubule assembly, or UPS dysfunction that are all modulated by LRRK2 in this A53T $\alpha$ synuclein mouse model $[86 \bullet \bullet]$. LRRK2 deletion appears to be protective in this A53T $\alpha$-synuclein model, thereby suggesting that LRRK2 inhibition could provide a potential strategy for preventing $\alpha$-synuclein-related neuronal toxicity. However, as a cautionary note, LRRK2 deletion in mice results in kidney and lung pathology that is characterized by impaired protein degradation pathways, increased apoptotic cell death, inflammatory responses, and abnormalities in the autophagy-lysosomal pathway [26-28]. LRRK2 could potentially modulate $\alpha$-synuclein-related neuropathology in transgenic mice by priming $\alpha$-synuclein aggregation or could act downstream to mediate $\alpha$-synuclein-induced neuronal toxicity. Alternatively, LRRK2 might inhibit $\alpha$ synuclein metabolism or turnover via the proteasomal and/ 
or lysosomal degradation pathways or could promote $\alpha$ synuclein protein translation. It will be important to clarify whether the effects of modulating LRRK2 expression in animal models are specific to $\alpha$-synuclein (or tau) or simply result from a global effect on protein turnover and/or translation.

LRRK2 mutations have been shown to promote inclusion formation in neural cell lines and cultured neurons through a kinase-dependent mechanism that could be correlated with cell death $[62,65]$. There is some variability in the morphology and frequency of LRRK2 inclusions with different mutations $[62,65]$. The exact nature or role of these inclusions is unclear. LRRK2 inclusion bodies may represent filamentous structures resulting from LRRK2 oligomerization that are surrounded by the cytoskeletal filament vimentin, stain positive for $\gamma$-tubulin, and can be disrupted by the microtubule polymerization inhibitor, nocodazole, suggesting that they are formed by active recruitment to the microtubule network or to cytoskeletal elements [65, 87]. In primary neurons, G2019S and I2020T LRRK2 form spheroid-like aggregates in neuronal processes that also contain hyperphosphorylated tau, swollen lysosomes, multivesicular bodies, distended vacuolated mitochondria, and disrupted cytoskeletal components [79]. In general, LRRK2 inclusions most likely result from structural or functional perturbations of the microtubule network and/or actin cytoskeleton, which may influence their assembly, dynamic organization, and vesicular transport. It is not clear whether these LRRK2 inclusions are required for LRRK2dependent neuronal degeneration. Furthermore, LRRK2 does not appear to form inclusions in idiopathic PD brains $[22,65,89]$, although a rigorous analysis of LRRK2 animal models and LRRK2 mutant PD brains awaits the development of improved antibodies.

LRRK2 has been shown to modulate the autophagy pathway. Impaired neurite outgrowth induced by G2019S LRRK2 is prevented by genetic inhibition of autophagy components but exacerbated by rapamycin, an activator of autophagy [82]. G2019S LRRK2 also promoted the accumulation of autophagic vacuoles in neuritic and somatic compartments of cultured neurons although it is not known whether this results from enhanced autophagy, impaired autophagic flux, or impaired fusion of autophagosomes with lysosomes [82]. LRRK2 localizes to autophagic vacuoles and multivesicular bodies in human brain and cultured human cells, while the overexpression of R1441C or G2019S LRRK2 in human cells promotes the accumulation of multivesicular bodies and large autophagic vacuoles containing incompletely digested material and increased levels of p62 $[20,30,79]$. In PD and dementia with Lewy bodies brains, LRRK2 abnormally accumulates in the endosomallysosomal compartment of neurons [89]. Impaired autophagy and endocytic vesicular trafficking are also evident in a yeast model expressing human LRRK2 and correlate with cellular toxicity [60]. The accumulation of autophagic vacuoles is also observed in the brains of aged G2019S and R1441C LRRK2 transgenic mice [71•]. The mechanisms underlying the effects of LRRK2 on the autophagy pathway are poorly understood. A recent study suggests that LRRK2 kinase activity may promote autophagy induction through activation of NAADP (nicotinic acid adenine dinucleotide phosphate) receptors, which mediate calcium efflux from lysosomes [91]. Despite substantial evidence demonstrating abnormal autophagy in LRRK2 disease models, what is not yet clear is whether autophagy is critically required for LRRK2dependent neurodegeneration. Future studies that activate or inhibit autophagy pathways in LRRK2 animal models with neurodegeneration will be important for clarifying the role of autophagy and exploring the therapeutic potential of this pathway.

\section{Mitochondria}

LRRK2 may regulate mitochondrial activity, morphology, and turnover [92]. LRRK2 localizes to the outer membrane of mitochondria in the mammalian brain [20]. The brains of aged G2019S LRRK2 transgenic mice reveal the abnormal accumulation of condensed and aggregated mitochondria consistent with altered mitochondrial autophagy (mitophagy) [71•]. G2019S LRRK2 expression also exacerbates structural and functional mitochondrial abnormalities that occur in neurons due to A53T $\alpha$-synuclein expression in transgenic mice $\left[86 \bullet^{\bullet}\right]$. In cultured fibroblasts derived from PD patients harboring the LRRK2 G2019S mutation, mitochondrial membrane potential and ATP levels are reduced whereas mitochondrial elongation and interconnectivity were increased, suggesting impaired mitochondrial function and morphology [93]. LRRK2 has recently been shown to regulate mitochondrial dynamics and function through a direct interaction with Drp1, a small GTPase required for mitochondrial fission [94]. LRRK2 mutations enhance mitochondrial fragmentation, reduce ATP levels, and increase reactive oxygen species in neurons through a Drp1dependent mechanism that may involve the recruitment of Drp1 to mitochondria via its interaction with LRRK2 [94]. Importantly, neuronal cell death induced by mutant LRRK2 could be prevented by inhibition of Drp1 or by promoting mitochondrial fusion via overexpression of mitofusin 2 [94], suggesting that inappropriate mitochondrial fission may underlie the neurotoxic effects of LRRK2. Therefore, impaired mitochondrial function and morphology may contribute to LRRK2-dependent neurodegeneration and it will be important to evaluate this pathway in LRRK2 animal models potentially by modulating mitochondrial fission and fusion. 


\section{Conclusions and Future Outlook}

Studies in cultured primary neurons and animal models have substantially advanced our understanding of the pathophysiology of LRRK2. Disease-associated mutations alter enzymatic activity in vitro but also induce phenotypes in cultured neurons in a kinase-dependent and occasionally a GTPasedependent manner. It will be important to clarify whether kinase inhibition represents a feasible strategy for attenuating LRRK2-dependent dopaminergic neurodegeneration, or related behavioral and pathological phenotypes, in currently available viral-based and transgenic animal models. A deeper understanding of the role of kinase activity in regulating LRRK2 activity, substrate phosphorylation, and associated downstream signaling pathways is of critical importance. Additional studies are still required to validate GTPase activity as a viable molecular target for inhibiting LRRK2 activity. At the molecular level, a number of intriguing pathways mostly familiar to PD research are emerging as potential candidates for an involvement in LRRK2related neuronal damage, including the microtubule network, actin cytoskeleton, vesicular trafficking, autophagy, mitochondria, protein degradation pathways, and translational control. The major challenge will be to determine which if any of these pathways are most important for mediating the pathogenic effects of LRRK2 mutations in neurons and validation of such mechanisms in appropriate LRRK2 animal models. A complete understanding of LRRK2 function and the pathogenic mechanisms of familial mutations will offer a number of opportunities for the identification of novel molecular targets that might prove useful for attenuating LRRK2-dependent neurodegeneration in PD.

Acknowledgments The authors are grateful for funding support from the Swiss National Science Foundation (grant no. 310030_127478), Michael J. Fox Foundation for Parkinson's Research, Parkinson Schweiz, NIH, NINDS (NS076160), EPFL, and Merck-Serono AG.

Disclosure No potential conflicts of interest relevant to this article were reported.

\section{References}

Papers of particular interest, published recently, have been highlighted as:

- Of importance,

-. Of major importance

1. Gasser T: Mendelian forms of Parkinson's disease. Biochim Biophys Acta 2009, 1792:587-596.

2. Lang AE, Lozano AM: Parkinson's Disease. First of Two Parts. N Engl J Med 1998, 339:1044-1053.
3. Lang AE, Lozano AM: Parkinson's disease. Second of Two Parts. N Engl J Med 1998, 339:1130-1143.

4. Paisán-Ruiz C, Jain S, Evans EW et al.: Cloning of the gene containing mutations that cause PARK8-linked Parkinson's Disease. Neuron 2004, 44:595-600.

5. Zimprich A, Biskup S, Leitner P et al.: Mutations in LRRK2 cause autosomal-dominant parkinsonism with pleomorphic pathology. Neuron 2004, 44:601-607.

6. Simon-Sanchez J, Schulte C, Bras JM et al.: Genome-wide association study reveals genetic risk underlying Parkinson's disease. Nat Genet 2009, 41:1308-1312.

7. Satake W, Nakabayashi Y, Mizuta I et al.: Genome-wide association study identifies common variants at four loci as genetic risk factors for Parkinson's disease. Nat Genet 2009, 41:13031307.

8. Biskup S, West AB: Zeroing in on LRRK2-linked pathogenic mechanisms in Parkinson's disease. Biochim Biophys Acta 2009, 1792:625-633.

9. Healy DG, Falchi M, O'Sullivan SS et al.: Phenotype, genotype, and worldwide genetic penetrance of LRRK2-associated Parkinson's disease: a case-control study. Lancet Neurol 2008, 7:583-590.

10. Giasson BI, Covy JP, Bonini NM et al.: Biochemical and pathological characterization of Lrrk2. Annals of Neurology 2006, 59:315-322.

11. Ross OA, Toft M, Whittle AJ et al.: Lrrk2 and Lewy body disease. Ann Neurol 2006, 59:388-393.

12. Wszolek ZK, Pfeiffer RF, Tsuboi Y et al.: Autosomal dominant parkinsonism associated with variable synuclein and tau pathology. Neurology 2004, 62:1619-1622.

13. Mata IF, Wedemeyer WJ, Farrer MJ et al.: LRRK2 in Parkinson's disease: protein domains and functional insights. Trends Neurosci 2006, 29:286-293.

14. Berger Z, Smith KA, LaVoie MJ: Membrane localization of LRRK2 is associated with increased formation of the highly active LRRK2 dimer and changes in its phosphorylation. Biochemistry 2010, 49:5511-5523.

15. Greggio E, Zambrano I, Kaganovich A et al.: The Parkinson Disease-associated Leucine-rich Repeat Kinase 2 (LRRK2) is a dimer that undergoes intramolecular autophosphorylation. J Biol Chem 2008, 283:16906-16914.

16. Deng J, Lewis PA, Greggio E et al.: Structure of the ROC domain from the Parkinson's disease-associated leucine-rich repeat kinase 2 reveals a dimeric GTPase. Proc Natl Acad Sci U S A 2008, 105:1499-1504.

17. Sen S, Webber PJ, West AB: Dependence of leucine-rich repeat kinase 2 (LRRK2) kinase activity on dimerization. J Biol Chem 2009, 284:36346-36356.

18. Jorgensen ND, Peng Y, Ho CCY et al.: The WD40 domain is required for LRRK2 neurotoxicity. PLoS One 2009, 4:e8463.

19. Biskup S, Moore D, Rea A et al.: Dynamic and redundant regulation of LRRK2 and LRRK1 expression. BMC Neurosci 2007, 8:102.

20. Biskup S, Moore DJ, Celsi F et al.: Localization of LRRK2 to membranous and vesicular structures in mammalian brain. Ann Neurol 2006, 60:557-569.

21. Galter D, Westerlund M, Carmine A et al.: LRRK2 expression linked to dopamine-innervated areas. Ann Neurol 2006, 59:714 719 .

22. Higashi S, Biskup S, West AB et al.: Localization of Parkinson's disease-associated LRRK2 in normal and pathological human brain. Brain Res 2007, 1155:208-219.

23. Higashi S, Moore DJ, Colebrooke RE et al.: Expression and localization of Parkinson's disease-associated leucine-rich repeat kinase 2 in the mouse brain. J Neurochem 2007, 100:368381. 
24. Westerlund M, Belin AC, Anvret A et al.: Developmental regulation of leucine-rich repeat kinase 1 and 2 expression in the brain and other rodent and human organs: Implications for Parkinson's disease. Neuroscience 2008, 152:429-436.

25. Piccoli G, Condliffe SB, Bauer M et al.: LRRK2 controls synaptic vesicle storage and mobilization within the recycling pool. J Neurosci 2011, 31:2225-2237.

26. Tong Y, Giaime E, Yamaguchi $\mathrm{H}$ et al.: Loss of leucine-rich repeat kinase 2 causes age-dependent bi-phasic alterations of the autophagy pathway. Mol Neurodegener 2012, 7:2.

27. Tong Y, Yamaguchi H, Giaime E et al.: Loss of leucine-rich repeat kinase 2 causes impairment of protein degradation pathways, accumulation of alpha-synuclein, and apoptotic cell death in aged mice. Proc Natl Acad Sci U S A 2010, 107:9879-9884.

28. Herzig MC, Kolly C, Persohn E et al.: LRRK2 protein levels are determined by kinase function and are crucial for kidney and lung homeostasis in mice. Hum Mol Genet 2011, 20:4209-4223.

29. Melrose H, Lincoln S, Tyndall G et al.: Anatomical localization of leucine-rich repeat kinase 2 in mouse brain. Neuroscience 2006, 139:791-794.

30. Alegre-Abarrategui J, Christian H, Lufino MMP et al.: LRRK2 regulates autophagic activity and localizes to specific membrane microdomains in a novel human genomic reporter cellular model. Hum Mol Genet 2009, 18:4022-4034.

31. Hatano $\mathrm{T}$, Kubo S, Imai $\mathrm{S}$ et al.: Leucine-rich repeat kinase 2 associates with lipid rafts. Hum Mol Genet 2007, 16:678-690.

32. Dodson MW, Zhang T, Jiang C et al.: Roles of the Drosophila LRRK2 homolog in Rab7-dependent lysosomal positioning. Hum Mol Genet 2011, Dec 30, Epub.

33. Gloeckner CJ, Schumacher A, Boldt K, Ueffing M: The Parkinson disease-associated protein kinase LRRK2 exhibits MAPKKK activity and phosphorylates MKK3/6 and MKK4/7, in vitro. J Neurochem 2009, 109:959-968.

34. Hsu CH, Chan D, Greggio E et al.: MKK6 binds and regulates expression of Parkinson's disease-related protein LRRK2. J Neurochem 2010, 112:1593-1604.

35. Carballo-Carbajal I, Weber-Endress S, Rovelli G et al.: Leucinerich repeat kinase 2 induces alpha-synuclein expression via the extracellular signal-regulated kinase pathway. Cell Signal 2010, 22:821-827.

36. West AB, Moore DJ, Choi $\mathrm{C}$ et al.: Parkinson's disease-associated mutations in LRRK2 link enhanced GTP-binding and kinase activities to neuronal toxicity. Hum Mol Genet 2007, 16:223-232.

37. Jaleel M, Nichols RJ, Deak $M$ et al.: LRRK 2 phosphorylates moesin at threonine-558: characterization of how Parkinson's disease mutants affect kinase activity. Biochem J 2007, 405:307-317.

38. West AB, Moore DJ, Biskup S et al.: Parkinson's diseaseassociated mutations in leucine-rich repeat kinase 2 augment kinase activity. Proc Natl Acad Sci U S A 2005, 102:16842-16847.

39. Nichols RJ, Dzamko N, Hutti JE et al.: Substrate specificity and inhibitors of LRRK2, a protein kinase mutated in Parkinson's disease. Biochem J 2009, 424:47-60.

40. Gillardon F: Leucine-rich repeat kinase 2 phosphorylates brain tubulin-beta isoforms and modulates microtubule stability-a point of convergence in Parkinsonian neurodegeneration? J Neurochem 2009, 110:1514-1522.

41. Kanao T, Venderova K, Park DS et al.: Activation of FoxO by LRRK2 induces expression of proapoptotic proteins and alters survival of postmitotic dopaminergic neuron in Drosophila. Hum Mol Genet 2010, 19:3747-3758.

42. Imai Y, Gehrke S, Wang HQ et al.: Phosphorylation of 4E-BP by LRRK2 affects the maintenance of dopaminergic neurons in Drosophila. EMBO J 2008, 27:2432-2443.

43. Qing H, Wong W, McGeer EG, McGeer PL: Lrrk2 phosphorylates alpha synuclein at serine 129: Parkinson disease implications. Biochem Biophys Res Commun 2009, 387:149-152.
44. Lin C-H, Tsai P-I, Wu R-M, Chien C-T: LRRK2 G2019S mutation induces dendrite degeneration through mislocalization and phosphorylation of Tau by recruiting autoactivated GSK3 \{beta\}. J Neurosci 2010, 30:13138-13149.

45. Kumar A, Greggio E, Beilina A et al.: The Parkinson's disease associated LRRK2 exhibits weaker in vitro phosphorylation of 4EBP compared to autophosphorylation. PLoS One 2010, 5:e8730.

46. Gloeckner CJ, Boldt K, von Zweydorf F et al.: Phosphopeptide analysis reveals two discrete clusters of phosphorylation in the Nterminus and the Roc domain of the Parkinson-disease associated protein kinase LRRK2. J Proteome Res 2010, 9:1738-1745.

47. Greggio E, Taymans JM, Zhen EY et al.: The Parkinson's disease kinase LRRK2 autophosphorylates its GTPase domain at multiple sites. Biochem Biophys Res Commun 2009, 389:449-454.

48. Webber PJ, Smith AD, Sen S et al.: Autophosphorylation in the leucine-rich repeat kinase 2 (LRRK2) GTPase domain modifies kinase and GTP-binding activities. J Mol Biol 2011, 412:94-110.

49. Kamikawaji $\mathrm{S}$, Ito G, Iwatsubo T: Identification of the autophosphorylation sites of LRRK2. Biochemistry 2009, 48:1096310975.

50. Li X, Tan YC, Poulose $\mathrm{S}$ et al.: Leucine-rich repeat kinase 2 (LRRK2)/PARK8 possesses GTPase activity that is altered in familial Parkinson's disease R1441C/G mutants. J Neurochem 2007, 103:238-247.

51. Lewis PA, Greggio E, Beilina A et al.: The R1441C mutation of LRRK2 disrupts GTP hydrolysis. Biochem Biophys Res Commun 2007, 357:668-671.

52. Ito G, Okai T, Fujino $G$ et al.: GTP binding is essential to the protein kinase activity of LRRK2, a causative gene product for familial Parkinson's disease $†$. Biochemistry 2007, 46:1380-1388.

53. Smith WW, Pei Z, Jiang $H$ et al.: Kinase activity of mutant LRRK2 mediates neuronal toxicity. Nat Neurosci 2006, 9:1231-1233.

54. Taymans JM, Vancraenenbroeck R, Ollikainen P et al.: LRRK2 kinase activity is dependent on LRRK2 GTP binding capacity but independent of LRRK2 GTP binding. PLoS One 2011, 6:e23207.

55. Haebig K, Gloeckner CJ, Miralles MG et al.: ARHGEF7 (BetaPIX) acts as guanine nucleotide exchange factor for leucine-rich repeat kinase 2. PLoS One 2010, 5:e13762.

56. Stafa K, Trancikova A, Webber P et al.: GTPase activity and neuronal toxicity of Parkinson's disease-associated LRRK2 is regulated by ArfGAP1. PLoS Genet 2012, 8:e1002527.

57. Moore DJ: The biology and pathobiology of LRRK2: Implications for Parkinson's disease. Parkinsonism Relat Disord 2008, 14:S92S98.

58. Gloeckner CJ, Kinkl N, Schumacher A et al.: The Parkinson disease causing LRRK2 mutation I2020T is associated with increased kinase activity. Hum Mol Genet 2006, 15:223-232.

59. Greggio E, Cookson MR: Leucine-rich repeat kinase 2 mutations and Parkinson's disease: three questions. ASN Neuro $2009,1$.

60. Xiong Y, Coombes CE, Kilaru A et al.: GTPase activity plays a key role in the pathobiology of LRRK2. PLoS Genet 2010, 6: e1000902.

61. Daniels V, Vancraenenbroeck R, Law BM et al.: Insight into the mode of action of the LRRK2 Y1699C pathogenic mutant. J Neurochem 2011, 116:304-315.

62. Smith WW, Pei Z, Jiang $H$ et al.: Leucine-rich repeat kinase 2 (LRRK2) interacts with parkin, and mutant LRRK2 induces neuronal degeneration. Proc Natl Acad Sci U S A 2005, 102:1867618681.

63. Ramsden N, Perrin J, Ren Z et al.: Chemoproteomics-based design of potent LRRK2-selective lead compounds that attenuate Parkinson's disease-related toxicity in human neurons. ACS Chem Biol 2011, 6:1021-1028.

64. - Lee BD, Shin J-H, VanKampen J et al.: Inhibitors of leucine-rich repeat kinase-2 protect against models of Parkinson's disease. Nat 
Med 2010, 16:998-1000. This study validated in vivo that dopaminergic neuronal degeneration induced by G2019S LRRK2 is kinase-dependent and can be attenuated by pharmacological kinase inhibition.

65. Greggio E, Jain S, Kingsbury A et al.: Kinase activity is required for the toxic effects of mutant LRRK2/dardarin. Neurobiol Dis 2006, 23:329-341.

66. Liu Z, Wang X, Yu Y et al.: A Drosophila model for LRRK2-linked parkinsonism. Proc Natl Acad Sci U S A 2008, 105:2693-2698.

67. Liu Z, Hamamichi S, Lee BD et al.: Inhibitors of LRRK2 kinase attenuate neurodegeneration and Parkinson-like phenotypes in Caenorhabditis elegans and Drosophila Parkinson's disease models. Hum Mol Genet 2011, 20:3933-3942.

68. $\mathrm{Ng} \mathrm{CH}$, Mok SZ, Koh $\mathrm{C}$ et al.: Parkin protects against LRRK2 G2019S mutant-induced dopaminergic neurodegeneration in Drosophila. J Neurosci 2009, 29:11257-11262.

69. - Dusonchet J, Kochubey O, Stafa K et al.: A Rat Model of Progressive Nigral Neurodegeneration Induced by the Parkinson's Disease-Associated G2019S Mutation in LRRK2. J Neurosci 2011, 31:907-912. This describes an adenoviral-based LRRK2 rat model that exhibits robust and progressive nigral dopaminergic neurodegeneration and tau pathology induced by G2019S LRRK2.

70. Deng X, Dzamko N, Prescott A et al.: Characterization of a selective inhibitor of the Parkinson's disease kinase LRRK2. Nat Chem Biol 2011, 7:203-205.

71. - Ramonet D, Daher JPL, Lin BM et al.: Dopaminergic Neuronal Loss, Reduced Neurite Complexity and Autophagic Abnormalities in Transgenic Mice Expressing G2019S Mutant LRRK2. PLoS One 2011, 6:e18568. This reports a transgenic mouse model expressing G2019S LRRK2 displaying late-onset dopaminergic neuronal degeneration and autophagic abnormalities.

72. - Li Y, Liu W, Oo TF et al.: Mutant LRRK2(R1441G) BAC transgenic mice recapitulate cardinal features of Parkinson's disease. Nat Neurosci 2009, 12:826-828. This is the first transgenic mouse model of LRRK2 reported that develops age-dependent and levodopa-responsive motor deficits, impaired dopaminergic neurotransmission, axonal pathology, and abnormal tau hyperphosphorylation.

73. Li X, Patel JC, Wang J et al.: Enhanced striatal dopamine transmission and motor performance with LRRK2 overexpression in mice is eliminated by familial Parkinson's disease mutation G2019S. J Neurosci 2010, 1788-1797.

74. Melrose HL, Dächsel JC, Behrouz B et al.: Impaired dopaminergic neurotransmission and microtubule-associated protein tau alterations in human LRRK2 transgenic mice. Neurobiol Dis 2010, 40:503-517.

75. Tong Y, Pisani A, Martella G et al.: R1441C mutation in LRRK2 impairs dopaminergic neurotransmission in mice. Proc Natl Acad Sci U S A 2009, 106:14622-14627.

76. Shin N, Jeong H, Kwon J et al.: LRRK2 regulates synaptic vesicle endocytosis. Exp Cell Res 2008, 314:2055-2065.

77. Sakaguchi-Nakashima A, Meir JY, Jin Y et al.: LRK-1, a C. elegans PARK8-Related Kinase, Regulates Axonal-Dendritic Polarity of SV Proteins. Curr Biol 2007, 17:592-598.

78. Iaccarino C, Crosio C, Vitale $\mathrm{C}$ et al.: Apoptotic mechanisms in mutant LRRK2-mediated cell death. Hum Mol Genet 2007, 16:1319-1326.
79. MacLeod D, Dowman J, Hammond R et al:: The familial Parkinsonism gene LRRK2 regulates neurite process morphology. Neuron 2006, 52:587-593.

80. Ho CC-Y, Rideout HJ, Ribe E et al.: The Parkinson disease protein leucine-rich repeat kinase 2 transduces death signals via fasassociated protein with death domain and caspase- 8 in a cellular model of neurodegeneration. J Neurosci 2009, 29:1011-1016.

81. Conde C, Caceres A: Microtubule assembly, organization and dynamics in axons and dendrites. Nat Rev Neurosci 2009, 10:319-332.

82. Plowey ED, Cherra SJ, Liu Y-J, Chu CT: Role of autophagy in G2019S-LRRK2-associated neurite shortening in differentiated SH-SY5Y cells. J Neurochem 2008, 105:1048-1056.

83. - Parisiadou L, Xie C, Cho HJ et al.: Phosphorylation of ezrin/ radixin/moesin proteins by LRRK2 promotes the rearrangement of actin cytoskeleton in neuronal morphogenesis. J Neurosci 2009, 29:13971-13980. This study describes a potential mechanism underlying neurite outgrowth defects induced by G2019S LRRK2 based upon rearrangement of the actin cytoskeleton.

84. Chan D, Citro A, Cordy JM et al.: Rac1 protein rescues neurite retraction caused by $\mathrm{G} 2019 \mathrm{~S}$ leucine-rich repeat kinase 2 (LRRK2). J Biol Chem 2011, 286:16140-16149.

85. Gandhi PN, Wang X, Zhu X et al.: The Roc domain of leucine-rich repeat kinase 2 is sufficient for interaction with microtubules. J Neurosci Res 2008, 86:1711-1720.

86. •• Lin X, Parisiadou L, Gu X-L et al.: Leucine-Rich Repeat Kinase 2 Regulates the Progression of Neuropathology Induced by Parkinson's-Disease-Related Mutant [alpha]-synuclein. Neuron 2009, 64:807-827. This study reports a functional interaction between LRRK2 and $\alpha$-synuclein in vivo in transgenic mice that could potentially explain the development of Lewy body pathology in LRRK2 PD brains.

87. Kett LR, Boassa D, Ho CC-Y et al.: LRRK2 Parkinson disease mutations enhance its microtubule association. Hum Mol Genet 2012, 21:890-899.

88. Rajput A, Dickson DW, Robinson CA et al.: Parkinsonism, Lrrk2 G2019S, and tau neuropathology. Neurology 2006, 67:1506-1508.

89. Higashi S, Moore DJ, Yamamoto R et al.: Abnormal localization of leucine-rich repeat kinase 2 to the endosomal-lysosomal compartment in lewy body disease. J Neuropathol Exp Neurol 2009, 68:994-1005.

90. Lichtenberg M, Mansilla A, Zecchini VR et al.: The Parkinson's disease protein LRRK2 impairs proteasome substrate clearance without affecting proteasome catalytic activity. Cell Death Dis 2011, 2:e196.

91. Gómez-Suaga P, Luzón-Toro B, Churamani D et al.: Leucine-rich repeat kinase 2 regulates autophagy through a calcium-dependent pathway involving NAADP. Hum Mol Genet 2011, 21:511-525.

92. Trancikova A, Tsika E, Moore DJ: Mitochondrial Dysfunction in Genetic Animal Models of Parkinson's Disease. Antioxid Redox Signal 2011, Oct 4, Epub.

93. Mortiboys H, Johansen KK, Aasly JO, Bandmann O: Mitochondrial impairment in patients with Parkinson disease with the G2019S mutation in LRRK2. Neurology 2010, 75:2017-2020.

94. Wang X, Yan MH, Fujioka $\mathrm{H}$ et al.: LRRK2 Regulates Mitochondrial Dynamics and Function through Direct Interaction with DLP1. Hum Mol Genet 2012, Jan 6, Epub. 